\title{
The effects of MyD88 deficiency on disease phenotype in dysferlin-deficient $A / J$ mice: role of endogenous TLR ligands
}

\author{
Kitipong Uaesoontrachoon,' Hee-Jae Cha,',2 Beryl Ampong,' Arpana Sali,' Jack Vandermeulen,' Benjamin Wei, \\ Brittany Creeden,' Tony Huynh,1,3 James Quinn,' Kathleen Tatem,' Sree Rayavarapu,1,4 Eric P Hoffman',4 and \\ Kanneboyina Nagaraju, ${ }^{l, 4, *}$ \\ Research Center for Genetic Medicine, Children's National Medical Center, Washington, DC, USA \\ ${ }^{2}$ Department of Parasitology and Genetics, Kosin University College of Medicine, Amnam-dong, Seo-gu, Busan, South Korea \\ 3 Endocrine Research Unit and the Australian National University Medical School, The Canberra Hospital, Australian Capital Territory, Australia \\ ${ }^{4}$ Department of Integrative Systems Biology, George Washington University School of Medicine and Health Sciences, Washington, DC, USA
}

*Correspondence to: Kanneboyina Nagaraju, DVM, PhD, Research Center for Genetic Medicine, Children's National Medical Center, I I I Michigan Avenue NW, Washington, DC 2001 0, USA. e-mail: knagaraju@cnmcresearch.org

\begin{abstract}
An absence of dysferlin leads to activation of innate immune receptors such as Toll-like receptors (TLRs) and skeletal muscle inflammation. Myeloid differentiation primary response gene 88 (MyD88) is a key mediator of TLR-dependent innate immune signalling. We hypothesized that endogenous TLR ligands released from the leaking dysferlin-deficient muscle fibres engage TLRs on muscle and immune cells and contribute to disease progression. To test this hypothesis, we generated and characterized dysferlin and MyD88 double-deficient mice. Double-deficient mice exhibited improved body weight, grip strength, and maximum muscle contractile force at 6-8 months of age when compared to MyD88-sufficient, dysferlin-deficient A/J mice. Double-deficient mice also showed a decrease in total fibre number, which contributed to the observed increase in the number of central nuclei/fibres. These results indicate that there was less regeneration in the double-deficient mice. We next tested the hypothesis that endogenous ligands, such as single-stranded ribonucleic acids (ssRNAs), released from damaged muscle cells bind to TLR-7/8 and perpetuate the disease progression. We found that injection of ssRNA into the skeletal muscle of pre-symptomatic mice ( 2 months old) resulted in a significant increase in degenerative fibres, inflammation, and regenerating fibres in A/J mice. In contrast, characteristic histological features were significantly decreased in double-deficient mice. These data point to a clear role for the TLR pathway in the pathogenesis of dysferlin deficiency and suggest that TLR-7/8 antagonists may have therapeutic value in this disease.

Copyright $\odot 2013$ Pathological Society of Great Britain and Ireland. Published by John Wiley \& Sons, Ltd.
\end{abstract}

Keywords: Toll-like receptor; dysferlin; single-stranded RNA; regeneration; degeneration; inflammation; osteopontin

Received 19 January 2013; Revised 2 April 2013; Accepted 22 April 2013

No conflicts of interest were declared.

\section{Introduction}

Defects caused by mutations in the gene encoding the skeletal muscle protein dysferlin lead to a group of muscle degenerative disorders known collectively as dysferlinopathies. These disorders include limb girdle muscular dystrophy type 2B (LGMD 2B), Miyoshi myopathy (MM), and distal anterior myopathy [1-4].

Dysferlin is a $230 \mathrm{kDa}$ transmembrane protein that is predominantly expressed in skeletal muscle but also found abundantly in tissues such as heart, kidney, and placenta [3]. We and other groups have also found dysferlin to be expressed in monocytes and macrophages [5-7]. Immunohistochemical staining of human and mouse tissues has localized dysferlin to the sarcolemma, T-tubules, and cytoplasmic vesicles.
Dysferlin is believed to play a role in maintaining and repairing injured membranes through the resealing of membrane lesions [8-13]. The absence of dysferlin leads to defective repair after membrane damage, often triggering cell death $[11,12]$. However, these changes alone do not explain the full clinical picture of dysferlinopathy. Multiple lines of evidence suggest that muscle inflammation may play an earlier and more dominant role in dysferlinopathies compared with other dystrophies. First, dysferlinopathy patients can have a sub-acute presentation, with about $25 \%$ of patients initially diagnosed with polymyositis [14-17]. Monocyte function in patients has been shown to be defective, with a pro-inflammatory phenotype [7]. Muscle histology shows active and early involvement of complement, membrane-attack complex, and TLR $[13,18]$. In muscle biopsies from dysferlin-deficient 
patients, the presence of mononuclear cell infiltrates, cytokine and chemokine components, and increased NF- $\kappa \mathrm{B}$ signalling are all indicative of an ongoing chronic inflammatory response with innate immune involvement that may be more aggressive than other dystrophies [13,19-21].

Osteopontin (OPN) is a pleiotropic pro-inflammatory cytokine expressed in a diverse range of tissues [22,23] and has been found to be significantly elevated in dystrophic skeletal muscle [24-26]. Osteopontin can exist in two forms: intracellular OPN (iOPN) or secreted OPN (sOPN). Intracellular OPN has been demonstrated to participate in TLR signalling by interacting with MyD88 and enhancing IFN- $\alpha$ production by plasmacytoid dendritic cells [27]. Immune cells such as macrophages constitutively expressed high levels of iOPN, whereas $\mathrm{T}$ cells express high levels of sOPN [27-29]. The principal sources of OPN in dystrophic muscle have been suggested to be from activated macrophages and T-lymphocytes [24]. The presence of activated macrophages in dysferlin-deficient muscles supports a potential role for OPN in the promotion of an inflammatory response.

It is typically challenging to dissect cause/effect relationships in myofibre defects and inflammatory responses in generating myofibre necrosis and muscle wasting. One effective approach is to delete specific components of the immune pathways in the dysferlindeficient mouse model and determine the effect of this on the phenotype of the double knockouts. This has been done for complement (C3, C5), T, and B cells. Complement was shown to regulate TLR-mediated inflammation [30], whereas dendritic cells (DCs), which express TLRs, are activated by TLR ligands to elicit T- and B-cell responses $[31,32]$. The lack of complement and $\mathrm{T}$ and $\mathrm{B}$ cells has been shown to ameliorate muscle pathology in the dysferlin-deficient mouse model and improve muscle strength in patients with dysferlin-deficient muscular dystrophy [33-35]. These results suggest that TLR-mediated muscle injury is the central mechanism in the pathogenesis of dysferlinopathy. We have previously shown that increased TLR expression results in an up-regulation and activation of inflammasomes in the skeletal muscle of dysferlindeficient mice [18]. Here we hypothesized that the activation of TLR-7/8 by nucleic acid [RNAs] from the damaged cells via the MyD88 pathway may play a role in the pathogenesis of dysferlinopathy through an increase in the recruitment of immune cells and subsequent muscle damage. To examine the role of TLRs in the pathogenesis of dysferlin deficiency, we generated dysferlin-deficient mice that are both MyD88-sufficient and MyD88-deficient and studied the effects of exogenous single-stranded RNA on muscle inflammation. We demonstrated that the MyD88-dependent TLR pathway is a critical player in the pathogenesis of dysferlin deficiency.

\section{Materials and methods}

\section{Animals}

Mice were handled according to local Institutional Animal Care and Use Committee guidelines. Dysferlin-deficient male A/J mice (JAX number 000646), an inbred strain bearing an ETn retrotransposon $(5-6 \mathrm{~kb})$ insertion in intron 4 of the dysferlin gene [36], were purchased from the Jackson Laboratory (Bar Harbor, ME, USA). Wild-type (WT) A/JOlaHsd male mice were purchased from the Harlan Laboratory (Indianapolis, IN, USA) and used as normal control mice. MyD88-deficient mice were kindly provided by Dr Shizuo Akira (Osaka University, Osaka, Japan). Double deficient mice (dysferlin-deficient; MyD88deficient) are generated as described in Supplementary Materials and Methods and Supplementary Figure 1.

\section{Real-time PCR}

Total RNA was extracted from the quadriceps muscles of non-injected 2-month-old and 6- to 8-month-old WT, A/J, and double-deficient mice (MyD88-deficient, dysferlin-deficient $\mathrm{A} / \mathrm{J}$ mice). cDNAs were synthesized and subjected to real-time Taqman PCR assays using an Applied Biosystems Prism 7900 HT (Applied Biosystems, Foster City, CA, USA). The following default ABI prism $7900 \mathrm{HT}$ amplification conditions were used: $2 \mathrm{~min}$ at $50{ }^{\circ} \mathrm{C}$ and $10 \mathrm{~min}$ at $95^{\circ} \mathrm{C}$, followed by 40 cycles of $15 \mathrm{~s}$ at $95^{\circ} \mathrm{C}$ and $1 \mathrm{~min}$ at $60{ }^{\circ} \mathrm{C}$. PCR primers for HPRT (Mm01545399), TLR-7 (Mm00446590), and TLR-8 (Mm04209873) were purchased from ABI (Foster City, CA, USA) and used at $1 \mu \mathrm{l}$ each per reaction. For osteopontin $(O P N)$ gene expression assays, a SYBR Green assay was used. SYBR Green master mix was purchased from ABI and used according to the manufacturer's instructions. We used primers for $O P N$ and the housekeeping gene $H P R T$ with the following sequences: OPN-F: CTTTACAGCCTGCACCCAGA and $O P N-R$ : GCCACAGAATGCTGTGTCCT; and HPRT-F: AGCCTAAGATGAGCGCAAGT and HPRT-R: TTACTAGGCAGATGGCCACA. These primers were used at $250 \mathrm{nM}$ per reaction, with the thermal profile mentioned above.

\section{Muscle functional tests}

We have followed the SOP for phenotyping developed by our group and TREAT NMD for NMD models (http://www.treat-nmd.eu/research/preclinical/ overview/). To compare muscle function between double-deficient and A/J mice, we assessed grip strength using a grip strength meter (GSM) as previously reported [37]. All the functional data were acquired in a manner blinded to the genotype of the animals. We also compared the in vitro force contractions of extensor digitorum longus (EDL) muscles isolated from both groups of mice as previously described [38]. In brief, EDL muscles were stimulated between two stainless steel plate electrodes with a 
voltage of single 0.2 -ms-square stimulation pulses that were steadily increased till supramaximal stimulation of the muscle was achieved at optimal length. At this optimal length, tetanic force contractions were obtained at increasing frequencies using 300-ms trains of pulses. After stimulation, the muscle length was measured with calipers to obtain the optimal fibre length for calculation of specific force.

\section{Histology}

Mice were euthanized via carbon dioxide inhalation, followed by cervical dislocation. Directly following euthanasia, quadriceps muscles were removed, embedded in OCT, snap-frozen in liquid nitrogen-cooled isopentane, and stored at $-80{ }^{\circ} \mathrm{C}$ for later processing. For histological analysis, quadriceps muscles cryosections $(10 \mu \mathrm{m}$ thick) were stained with haematoxylin and eosin (H\&E). Digital images were taken using an Eclipse E800 (Nikon, Japan) microscope, and blinded analysis was carried out using Image $\mathbf{J}(\mathrm{NIH})$ as previously reported [37].

\section{Muscle injury and muscle preparation}

Endotoxin-free single-stranded RNA (ssRNA; $30 \mu \mathrm{l}$ at $9 \mu \mathrm{g} / \mathrm{ml}$ in saline; InvivoGen, San Diego, CA, USA) was injected directly into the right tibialis anterior (TA) muscle of 2-month-old male WT, A/J or doubledeficient mice using a 27.5-gauge insulin syringe. The left TA muscle was injected with $30 \mu \mathrm{l}$ of saline to serve as an injection control. Three repeated injections were done over a period of 10 days, with a 3 -day recovery period between each injection. Muscles were harvested 2 days after the final injection.

\section{Osteopontin ELISA}

A 96-well-plate mouse osteopontin immunoassay was purchased from R\&D Systems (Minneapolis, MN, USA). The assay was performed according to the manufacturer's instructions.

\section{Immunohistochemical staining}

TA muscle sections (10 $\mu \mathrm{m}$ thick) were blocked for $1 \mathrm{~h}$ in PBS containing $10 \%$ sheep serum and then incubated overnight at $4{ }^{\circ} \mathrm{C}$ with either rat anti-mouse CD11b (1 : 25; eBiosciences, San Diego, CA, USA) or mouse anti-chicken embryonic myosin heavy chain (1: 50; Development Studies Hybridoma Bank, Iowa City, IA, USA) diluted in PBS with 2\% sheep serum. The following day, sections were washed three times in PBS and incubated with either Alexa Fluor-488 goat anti-rat IgG (1 : 250; Invitrogen, Carlsbad, CA, USA) or Alexa Fluor-488 goat anti-mouse $\operatorname{IgG}_{1}$ (1: 250; Invitrogen) for a further $1 \mathrm{~h}$ at room temperature. Sections were then washed three times with PBS and counterstained with DAPI. Slides were stored at $4{ }^{\circ} \mathrm{C}$ prior to imaging under fluorescent illumination using a Zeiss Aviovert inverted microscope (Zeiss, Thornwood, NY, USA).

\section{Statistical analysis}

All data are presented as means \pm standard error of the mean. Comparisons between two groups were made by unpaired Student's $t$-tests. $p<0.05$ was considered significant. Quantitative PCR results were analysed for significant differences by a 2000-sample pairwise fixed reallocation randomization test using REST software [39].

\section{Results}

\section{MyD88 deficiency improves the disease phenotype of dysferlin-deficient $A / J$ mice}

Significant muscle pathology in $\mathrm{A} / \mathrm{J}$ mice is not seen until 5-6 months of age [36]. To study disease onset and progression, we genotyped (Supplementary Materials and Methods and Supplementary Figure 1) and performed systematic phenotyping of MyD88-sufficient and -deficient A/J mice at the presymptomatic (2-month-old) and symptomatic (6- to 8month-old) stages and expressed the values as the percentage change relative to WT. We found a significant increase in body weight of $\mathrm{A} / \mathrm{J}$ mice compared with double-deficient mice at 2 months of age. However, by $6-8$ months of age, double-deficient mice showed a significant increase in body weight than that of $A / J$ mice (Figure 1A).

Analysis of the forelimb grip strength showed the same trend as the body weight, where the forelimb grip strength of $\mathrm{A} / \mathrm{J}$ mice was significantly stronger than that of double-deficient mice at 2 months of age. However, after the onset of disease at 6-8 months of age, the forelimb grip strength of double-deficient mice was significantly stronger than that of $\mathrm{A} / \mathrm{J}$ mice. With age, there was a decline in the forelimb strength in $\mathrm{A} / \mathrm{J}$ mice, whereas an improvement in the forelimb strength was observed in double-deficient mice (Figure 1B). Similarly, the hindlimb grip strength of doubledeficient mice was significantly higher than that of A/J mice (data not shown). However, grip strength measurements normalized to body weight yielded no significant differences in either the forelimb or the hindlimb at either time point (data not shown).

Next, we assessed the specific force and maximum contractile force of EDL muscles and found only the maximum contractile force to be significantly higher in double-deficient EDL than in A/J EDL muscles of 6- to 8-month-old mice. With age, the specific force stabilized and the maximum contractile force increased in the double-deficient muscles. However, A/J muscles showed a significant decrease in both maximal and specific EDL contractile force with age (Figures 1C and 1D), indicating loss of force with age.

\section{Mice with MyD88 deficiency show less muscle damage}

Histological examination of the H\&E-stained quadriceps muscle sections of 6- to 8-month-old mice 
A

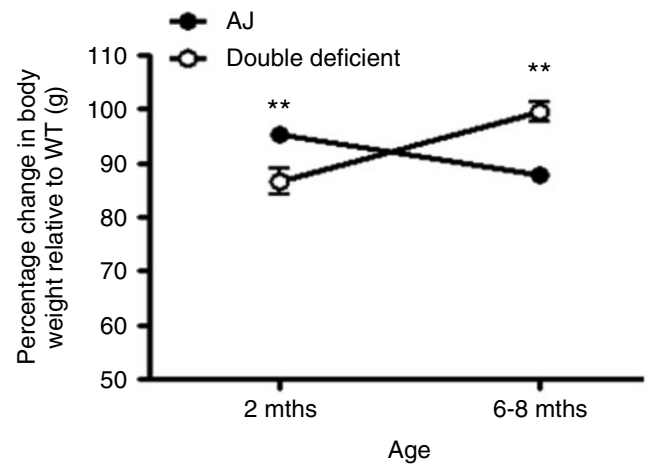

C

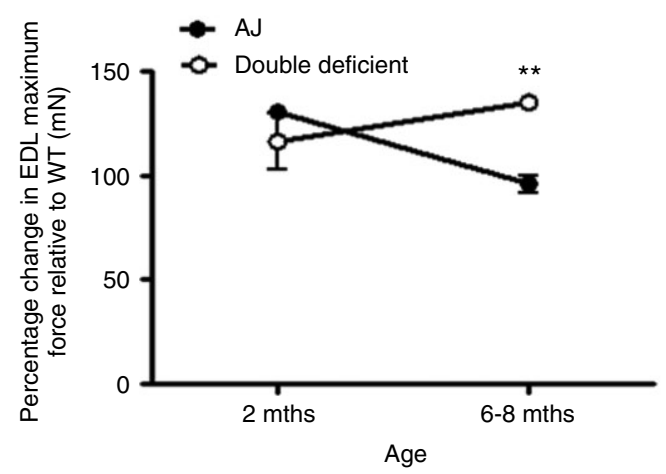

\section{B}

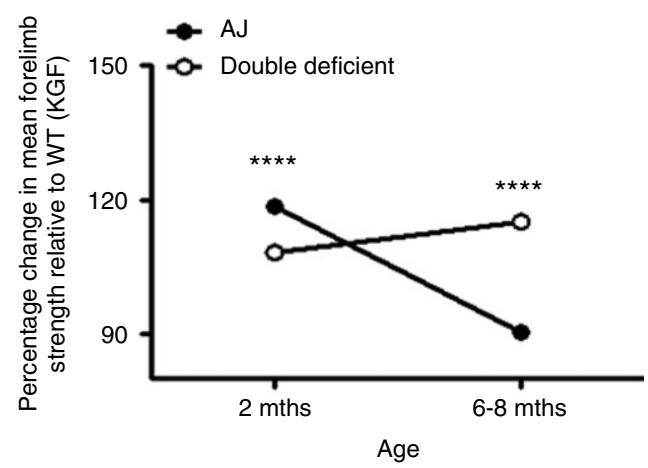

D

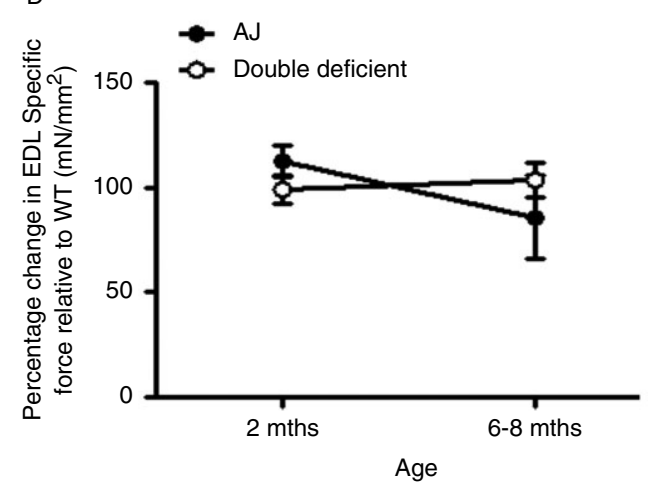

Figure 1. Improved body weight and muscle function in double-deficient mice. Body weight and muscle strength were compared at 2 months (pre-symptomatic) and 6-8 months (symptomatic) and expressed as percentage change relative to WT. Double-deficient mice showed improved body weight ( $A ; n=6$ \& 6 (A/J \& double deficient) at 2 months; $n=6(A / J)$ and $n=3$ (double deficient) at $6-8$ months), increased forelimb grip strength $(B ; n=14(A / J)$ \& 4 (double deficient) at 2 months; $n=6(A / J)$ and $n=3$ (double deficient) at 6-8 months), and maximum contractile force ( $\mathrm{C} ; \mathrm{n}=4 \mathrm{E} 3$ (A/J \& double deficient) at 2 and $6-8$ months) but no difference in in vitro specific force $(D ; n=4(A / J)$ \& 3 (double deficient) at 2 months; $n=6$ \&t 6 (A/J \& double deficient) at $6-8$ months) at 6 -8 months of age when compared to A/J mice. Data are expressed as means \pm S.E.M. ( $n=$ at least 3 animals from each genotypes). ${ }^{* *} p \leq 0.01$, ${ }^{* * *} p \leq 0.0001$; unpaired Student's t-test for comparisons within each age group.

Table 1. Comparison of histological parameters in the quadriceps muscles of A/J mice and double-deficient mice at 6-8 months of age

$\begin{array}{lrrr}\text { Measurement } & \mathrm{A} / \mathrm{J}(n=3) & \text { Double-deficient }(n=4) & p \text {-value } \\ \text { Total number of fibres per field } & 99.290 \pm 4.375 & 82.720 \pm 3.572 & 0.0059 \\ \text { Regenerating fibres per field } & 0.071 \pm 0.071 & 0.444 \pm 0.232 & 0.1791 \\ \text { Fibres with central nuclei per field } & 8.714 \pm 1.447 & 11.610 \pm 1.547 & 0.1921 \\ \text { Peripheral nuclei per fibre } & 1.453 \pm 0.041 & 1.480 \pm 0.059 & 0.7326 \\ \text { Central nuclei per fibre } & 0.107 \pm 0.017 & 0.185 \pm 0.028 & 0.0371 \\ \text { Total nuclei per fibre } & 1.561 \pm 0.043 & 1.664 \pm 0.067 & 0.2312 \\ \text { Ratio of central to peripheral nuclei per fibre } & 0.075 \pm 0.012 & 0.126 \pm 0.019 & 0.0362 \\ \text { Number of inflammatory foci per muscle* } & 34.000 \pm 4.509 & 25.500 \pm 6.185 & 0.3492\end{array}$

*A group of ten inflammatory cells was considered as one inflammatory foci.

revealed significantly lower total fibre numbers, with significantly higher numbers of central nuclei per fibre in the double-deficient compared with the A/J muscles. In addition, the ratio of central to peripheral nuclei per fibre was significantly higher in the double-deficient than in the A/J muscles. The increase in central nuclei per fibre in the double-deficient muscles was not surprising, given that these mice had a significantly lower total muscle fibre number than the $\mathrm{A} / \mathrm{J}$ mice. There was also a $25 \%$ reduction in the number of inflammatory foci per muscle in double-deficient compared with $\mathrm{A} / \mathrm{J}$ mice but this reduction was not statistically significant (Table 1). Taken together, these results suggest that the muscles of double-deficient mice underwent less damage than the $\mathrm{A} / \mathrm{J}$ muscles.
Effect of injection of an endogenous TLR ligand, ssRNA, on dysferlin-null and double-deficient mouse muscles

We have previously shown that TLRs are functionally active in A/J mice [18]. Quadriceps muscles from 2and 6- to 8-month-old WT, A/J and double deficient mice were subjected to real-time PCR analysis. Both targeted TLR-7 and TLR-8 were expressed but were not significantly altered at the pre-symptomatic period ( 2 months) between WT and A/J mice or WT and double-deficient muscles. However, TLR-7 was significantly up-regulated in A/J muscles compared with WT muscles at 6-8 months of age (symptomatic period), whereas TLR-7 was significantly down-regulated in 


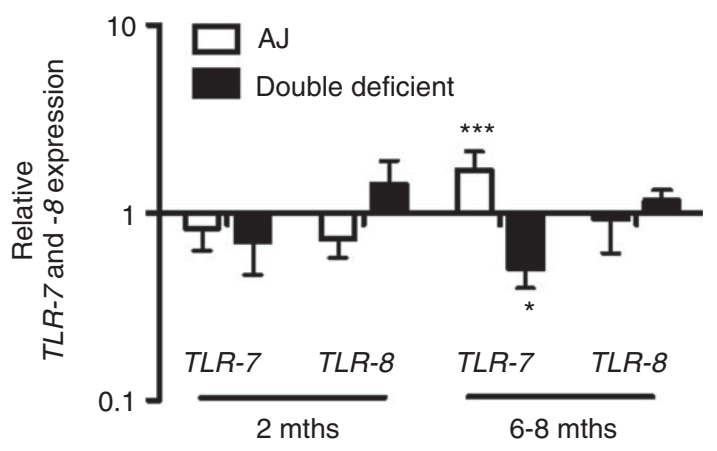

Figure 2. Analysis of $T L R-7$ and $T L R-8$ gene expression in noninjected muscles of WT, $A / J$, and double-deficient mice. cDNA was synthesized and used as a template for real-time PCR analysis. The $C_{\mathrm{t}}$ values were used to calculate $T L R-7$ and $T L R-8$ gene expression in $\mathrm{A} / \mathrm{J}$ and double deficient mice, normalized to $H P R T$, relative to WT mice at both 2 and 6-8 months. Data are presented as means \pm SEM ( $n=$ at least 3 animals). ${ }^{*} p \leq 0.05$ and ${ }^{* * *} p \leq 0.001$; the randomization reallocation test was used for comparison.

double-deficient muscles at this age. TLR- 8 was not significantly altered for either genotype compared with WT muscles at 6-8 months (Figure 2).

Next, we induced injury during the pre-symptomatic period (at 2 months of age) by intramuscular injections of either TLR-7/8 ligand (ssRNA) or saline into the TA muscles of WT, A/J, and double-deficient mice. This ssRNA was chosen to test the hypothesis that RNAs released from damaged or necrotic cells can activate TLR-7/8 and subsequently worsen the pathology. A microarray-based comparison (Supplementary Materials and Methods for illumina beadchip microarray) of the saline- and ssRNA-injected TA muscles of WT, $\mathrm{A} / \mathrm{J}$, and double-deficient mice showed that a total of 1159 genes (3.8\% of the total transcripts) were differentially expressed between ssRNA- and saline-injected $\mathrm{A} / \mathrm{J}$ muscles. Of the 1159 transcripts, 251 (21.7\%) were associated with inflammatory responses. Both targets for ssRNA, TLR-7 (fold change 5.8 and $p$ value 5.78E06 ) and $T L R-8$ (fold change 3.0 and $p$ value 5.9E-05), were up-regulated significantly in the ssRNA-treated $\mathrm{A} / \mathrm{J}$ muscles. Also significantly up-regulated in these muscles were $T L R-1,-2,-4,-5,-6$, and -13 as well as MyD88-associated signalling genes. Pathways activated by ssRNA in the A/J muscles were analysed by Ingenuity Pathway Analysis and are presented in Supplementary Figure 2 and Supplementary Tables 1 and 2.

In both the double-deficient and the WT mice, we did not detect any significant changes in the transcript profiles between ssRNA- and saline-injected muscles in the pathways for which we saw differences in A/J mice (Supplementary Figure 3), suggesting that these pathways are unaffected at the gene expression level in both genotypes.

\section{MyD88-deficient muscle shows a decrease in mononuclear cell infiltration in response to ssRNA injections}

The response of the TA muscles to the injected ssRNA during the pre-symptomatic period (at 2 months of
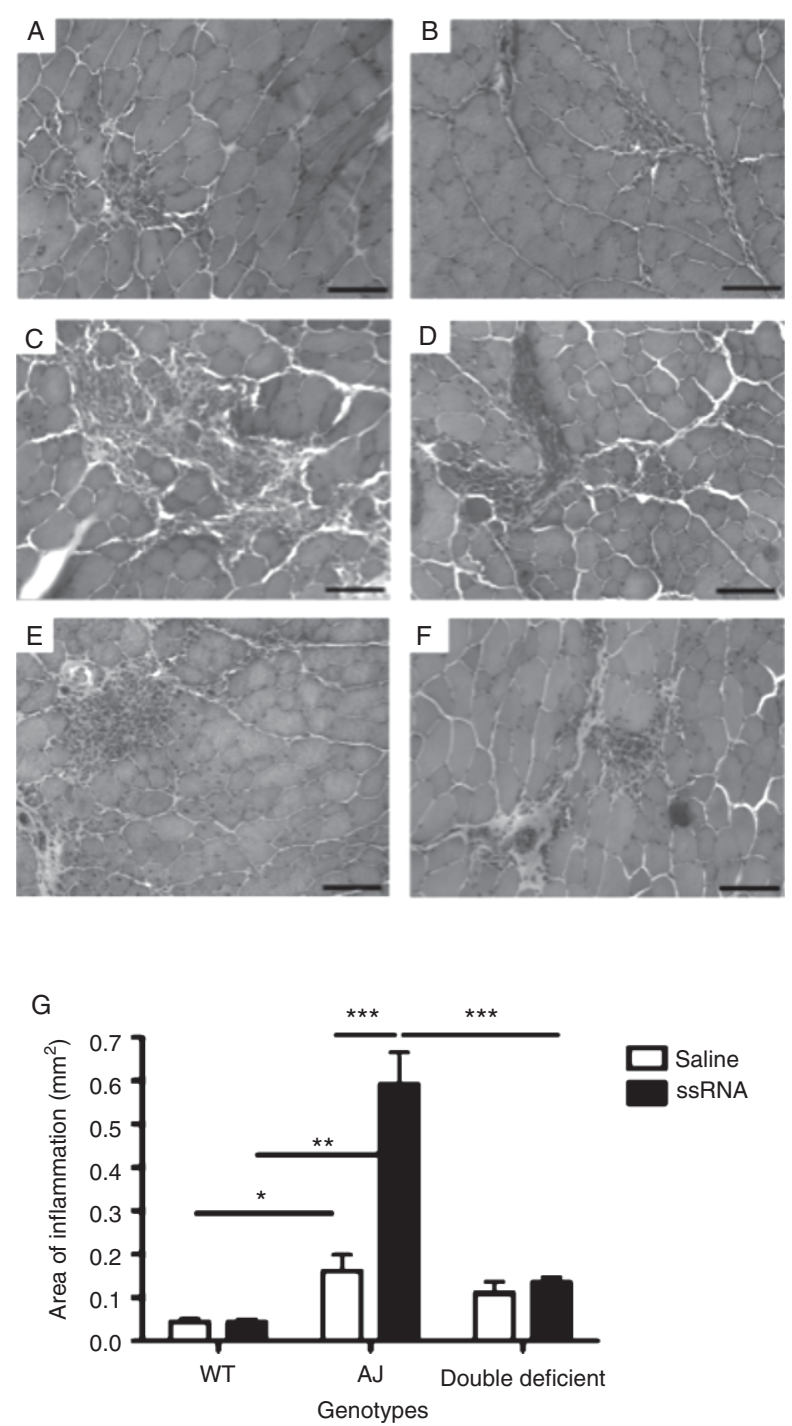

Figure 3. Quantitation of mononuclear cell infiltrates in the HEtE-stained sections of WT, A/J, and double-deficient mice. Representative H\&tE-stained transverse sections from ssRNAinjected (A: WT; $C: A / J$; E: double-deficient) and saline-injected (B: WT; D: A/J; F: double-deficient) TA muscles. (G) Quantitation of the inflammatory area in muscle sections injected with either ssRNA or saline in WT, $\mathrm{A} / \mathrm{J}$, and double-deficient mice. Data are expressed as means \pm SEM ( $n=4$ animals from each treatment and genotype). ${ }^{*} p \leq 0.05,{ }^{* *} p \leq 0.01$, and ${ }^{* * *} p \leq 0.001$; unpaired Student's $t$-test for comparisons indicated by lines above the bars. Bar $=200 \mu \mathrm{m}$.

age) was also examined histologically. Representative images of the H\&E-stained muscle sections showed normal muscle histology with no infiltration of cells in the non-injured muscles of either $\mathrm{A} / \mathrm{J}$ or doubledeficient mice (data not shown). There was a significant increase in mononuclear cell infiltration in $\mathrm{A} / \mathrm{J}$ muscles injected with ssRNA compared with saline (Figures $3 \mathrm{C}$ and $3 \mathrm{D})$. However, this response was significantly reduced in double-deficient (Figures $3 \mathrm{E}$ and $3 \mathrm{~F}$ ) and WT muscles (Figures 3A and 3B), with the mononuclear cells confined to the injection site in WT muscles (Figure 3G). These data suggest that TLR-7/8 ssRNA, through MyD88, mediates the influx of mononuclear cells into the muscles. There was no difference in 

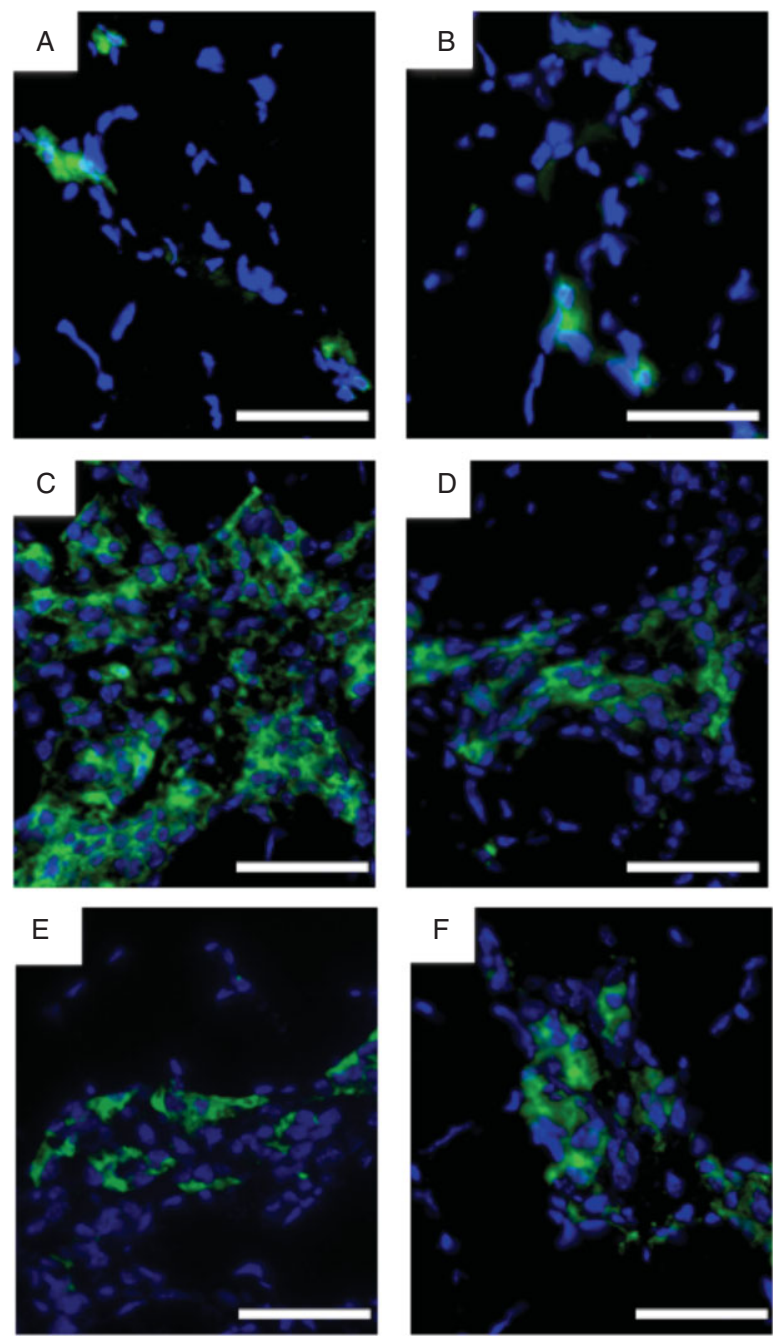

Figure 4. Immunohistochemical staining of the mononuclear cell infiltrate in ssRNA- or saline-treated muscles. Cryostat sections of muscles injected with ssRNA (A: WT; C: A/J; E: double-deficient) or saline (B: WT; D: A/J; F: double-deficient) were immunostained for CD11b (green) and counterstained with DAPI (blue). Bar $=200 \mu \mathrm{m}$.

the area of inflammation between the TA muscles of $\mathrm{A} / \mathrm{J}$ and double-deficient mice in the saline-injected muscles, since muscle inflammation is not typically seen at the pre-symptomatic stage.

To determine whether some of these mononuclear cells were inflammatory cells, we used immunohistochemical staining for CD11b, a marker for leukocytes, monocytes/macrophages, and natural killer cells, in the ssRNA- and saline-injected muscles of WT, $\mathrm{A} / \mathrm{J}$, and double-deficient mice. Staining of these muscles for CD11b showed an extensive number of CD11b-positive cells in the A/J ssRNA-treated group (Figure $4 \mathrm{C}$ ), but not in the $\mathrm{A} / \mathrm{J}$ saline-treated (Figure 4D), WT (Figures 4A and 4B), or double-deficient (Figures 4E and 4F) groups. These results further confirmed the findings of our H\&E analysis.

Up-regulation of the osteopontin gene and protein in ssRNA-treated dysferlin muscle

Because OPN is associated with inflammation in dystrophic muscle $[25,40,41]$, we investigated the
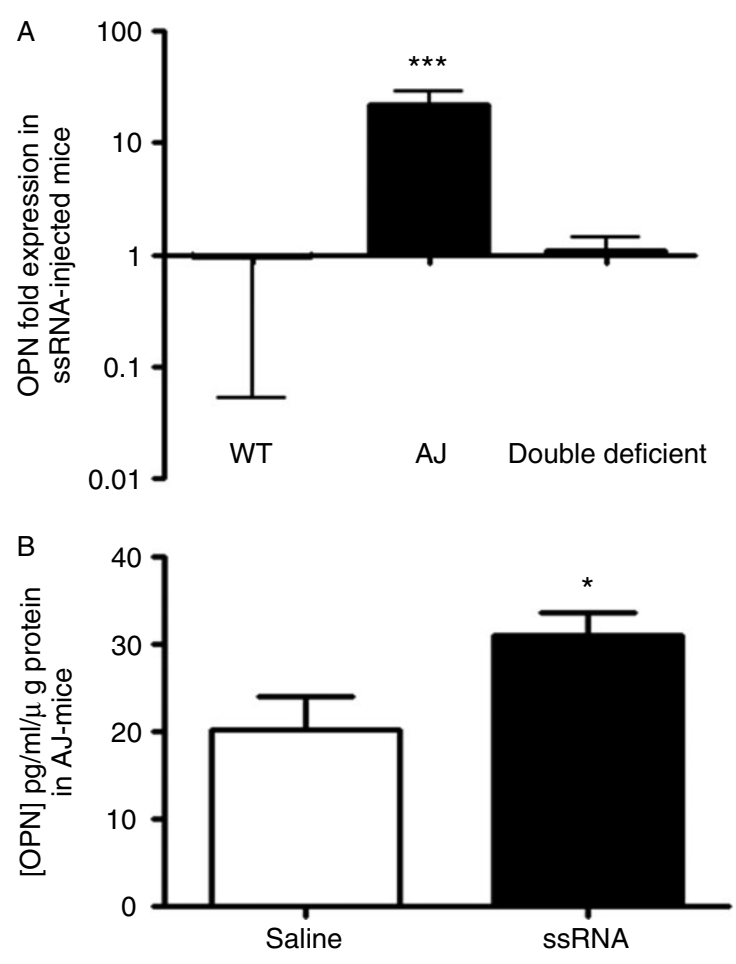

Figure 5. Analysis of OPN expression in muscles injected with either ssRNA or saline. (A) Real-time PCR was performed on cDNA synthesized from RNA extracted from muscle samples injected with either saline or ssRNA in WT, $A / J$, and double-deficient muscles. The $C_{t}$ values were used to calculate $O P N$ gene expression in ssRNA-treated muscles normalized to HPRT, relative to the normalized level of OPN expression in saline-treated muscles. (B) ELISA detection of OPN levels in saline- and ssRNA-injected muscles of A/J mice. Data are presented as means \pm SEM $(n=4$ animals from each treatment and genotype). ${ }^{*} p \leq 0.05$ and ${ }^{* *} p \leq 0.001$; the randomization reallocation test was used for comparison of ssRNA-treated and saline-treated muscles in A. Unpaired Student's $t$-test was used for comparisons in B.

regulation of $O P N$ expression in the injected skeletal muscle of WT, A/J, and double-deficient mice. Treatment of $\mathrm{A} / \mathrm{J}$ muscles with ssRNA resulted in a significant up-regulation of $O P N$ gene expression. However, this effect was not seen in double-deficient and WT muscles (Figure 5A). ELISA analysis of the injected muscles confirmed that ssRNA-stimulated A/J muscle showed an increase in the synthesis and secretion of OPN protein compared with saline-injected muscles (Figure 5B).

MyD88-deficient muscles show less muscle damage in response to ssRNA

Histomorphometric analysis of the H\&E-stained muscle sections revealed that ssRNA-injected A/J muscles exhibited higher numbers of degenerative muscle fibres than saline-injected muscles, consistent with the increase that we observed in inflammatory cell infiltration. There was no difference in the number of degenerative muscle fibres between the ssRNA- and saline-injected muscles in both double-deficient and WT mice. In addition, these values were significantly 

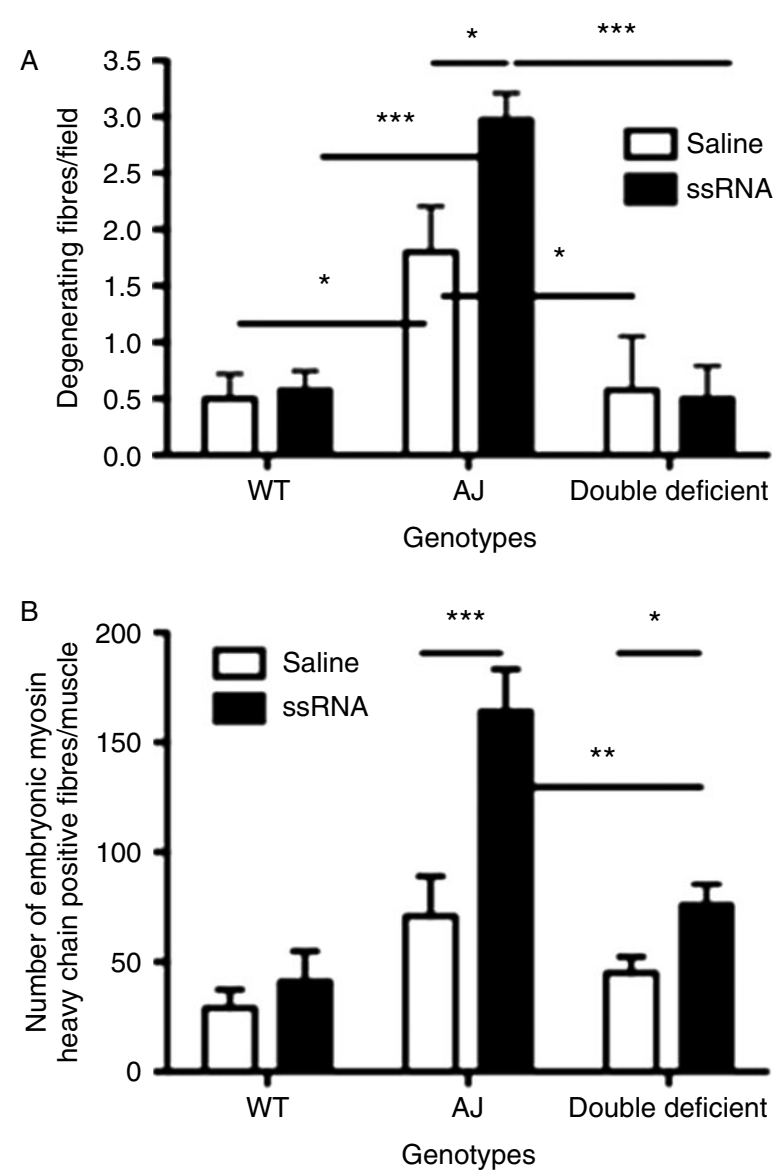

Figure 6. Histomorphometric analysis of the muscle sections injected with either saline or ssRNA. (A) Comparison of the number of degenerating fibres by counting the total number of degenerating fibres per field for muscles injected with either saline or ssRNA from each genotype (WT, A/J, and double-deficient mice). (B) Comparison of the number of embryonic myosin heavy chain-positive fibres per muscle between muscles injected with either saline or ssRNA for each genotype. Data are expressed as means \pm SEM $(n=4$ animals from each treatment and genotype). ${ }^{*} p \leq 0.05,{ }^{* *} p \leq 0.01$, and ${ }^{* * *} p$ $\leq 0.001$; unpaired Student's $t$-test was used.

lower in WT and double-deficient mice than in A/J mice (Figure 6A).

We next evaluated embryonic MHC-positive immature muscle fibres in the injected muscle sections of all three mouse strains. In $\mathrm{A} / \mathrm{J}$ mice, there was a significant increase in the number of fibres labelled with embryonic MHC in the ssRNA-injected muscles compared with the saline-injected mice, indicating that ssRNA treatment resulted in more muscle damage. However, there were significantly fewer embryonic MHC-positive fibres in the saline- and ssRNA-injected muscles in double-deficient mice compared with $\mathrm{A} / \mathrm{J}$ mice. In WT mice, there was no difference in the number of embryonic MHC-positive fibres between the ssRNA- and saline-injected muscles, and these numbers were also significantly lower than those of $\mathrm{A} / \mathrm{J}$ mice (Figure 6B). Taken together, these data indicated that the lack of MyD88 resulted in reduced myofibre damage, suggesting that the TLR pathway plays a significant role in myofibre inflammation and damage in dysferlinopathy.

\section{Discussion}

We have examined two aspects of the association between TLRs and the dysferlin-deficient disease phenotype. We first looked at the overall effects of the lack of MyD88 on a dysferlin-deficient A/J background. Characterization of double-deficient mice indicated that they had increased body weight and strength (grip strength and in vitro force) at 6-8 months of age, a time at which the disease has already begun, in comparison to $\mathrm{A} / \mathrm{J}$ mice. These mice also had more central nucleated muscle fibres and a significantly higher ratio of central to peripheral nuclei. However, the total fibre number was significantly lower in double-deficient mice than in $\mathrm{A} / \mathrm{J}$ mice. This most likely contributed to the higher number of centrally nucleated muscle fibres and the higher ratio of central to peripheral nuclei per muscle fibre that we observed in the double-deficient mice. The lower total number of muscle fibres may indicate less damage to the muscle fibres of doubledeficient mice, since more damage to the muscle leads to more regeneration and an increase in the total muscle fibre number, as seen in $\mathrm{A} / \mathrm{J}$ mice. Overall, the increased strength observed in double-deficient mice compared with $\mathrm{A} / \mathrm{J}$ mice could be due to less damage to the muscle fibres. The absence of MyD88 renders the TLR signalling inactive, leading to less cytokine production, inflammatory cell infiltration, and damage to the muscles, especially healthy bystander muscles $[42,43]$.

The second part of this study looked specifically at the effect of the activation of TLR-7 and -8 by endogenous ligand on the disease phenotype in dysferlinopathy. There is substantial evidence that the initiation signal to activate the TLRs can come from damaged or stressed cells in the absence of infection [44]. Studies have shown that loss of dysferlin results in a defect in the muscle membrane repair machinery, which prevents the resealing of the membrane after injury. This discontinuity of the membrane allows the entry of toxic agents such as oxidants, the release of intracellular molecules that produce an inflammatory response, and eventual cell death [11-13,45].

Damaged cells are known to release ribonucleic acids (RNAs) during the disease process [46,47]. We screened several endogenous TLR ligands such as HMGB-1, ssRNA, hyaluronic acid fragments, and lipopolysaccharide (LPS) in an in vitro system but found that only ssRNA elicited a significant response (data not shown). We therefore pursued the effect of ssRNA in the in vivo model. To investigate the role of ssRNAs in dysferlinopathy, we induced pathology by injecting ssRNA, a ligand for TLR-7 and -8, in WT, A/J, and double-deficient mice during the pre-symptomatic period. We selected 2-month-old pre-symptomatic mice for the injection studies because at this age, A/J muscles show no histological disease but both TLR-7 and TLR-8 are expressed. Injection injury coupled with the presence of exogenous ssRNA 


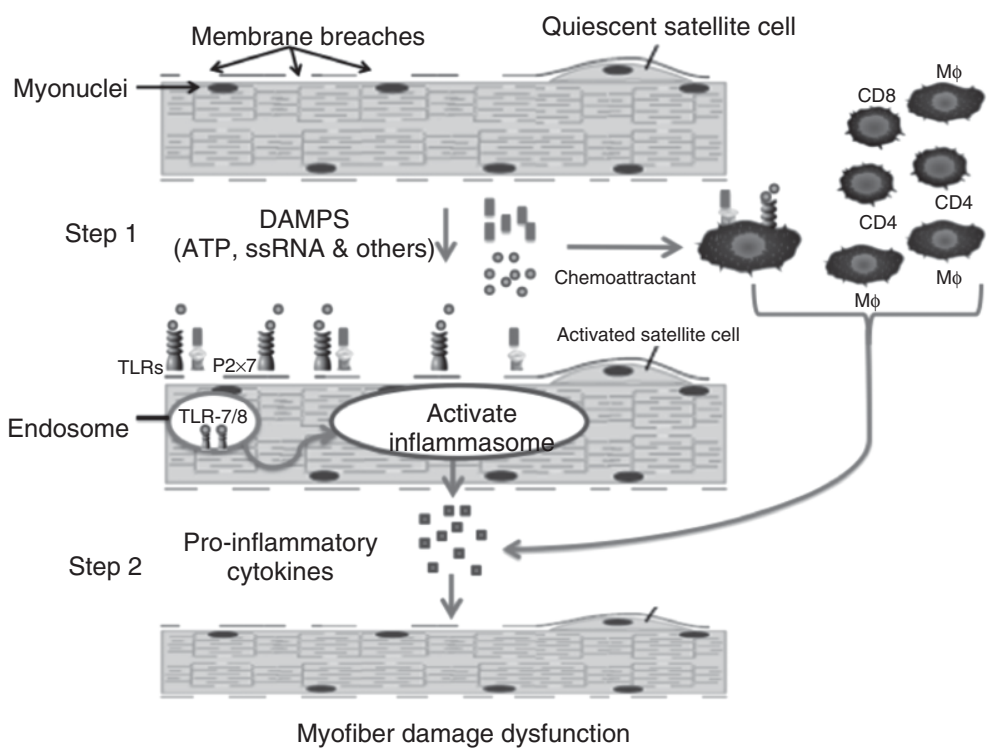

Figure 7. Membrane breaches in dysferlin-deficient skeletal muscle release damage-associated molecular pattern molecules (DAMPs), which include endogenous TLR ligands such as ssRNA and ATP. These ligands bind to their respective receptors (ssRNA to TLR-7/8 and ATP to P2x7 receptor; step 1). Signalling through these receptors activates the inflammasome pathway in skeletal muscle as well as immune cells, leading to the production of pro-inflammatory cytokines such as IL-1, TNF OPN (step 2). These cytokines activate immune cells and exacerbate the inflammatory response and damage to skeletal muscle, leading to a self-sustaining disease.

mimics the active muscle disease state. We demonstrated that ligands such as ssRNA can activate TLRs and thereby trigger an immune/inflammatory response. This response, in turn, can result in an exacerbation of the disease phenotype.

We have previously shown that myofibre membrane damage can activate TLR-7 in patients with Duchenne muscular dystrophy (DMD), thereby triggering an inflammatory response through the activation of NF$\kappa \mathrm{B}$ [48]. Here we have shown that not only TLR-7 and TLR-8, but also several other MyD88-dependent TLRs (TLR-1, -2, -4, -5, -6, and -13) are highly expressed as a result of damage to the skeletal muscle in $\mathrm{A} / \mathrm{J}$ mice. These results indicate that, in addition to RNA, there are likely other endogenous ligands that are released during the injury caused by injection of ssRNA into the skeletal muscle that would activate these TLRs. Therefore, the inflammatory response is a complex process and blocking a single pathway of the inflammatory network may not necessarily be beneficial in reducing the pathology.

Cells of the immune system such as macrophages and neutrophils have been shown to produce active metabolites that can promote inflammation and necrosis in muscle fibres [42,49-52]. The increase that we observed in the recruitment of inflammatory cells to the site of damage via activation of TLR-7 and -8 by ssRNA could be the same process that leads to the necrosis in $\mathrm{A} / \mathrm{J}$ muscle fibres. This possibility is further supported by the results of our experiments with double-deficient muscles. For example, these muscles have reduced inflammatory cell infiltration and lower numbers of degenerating and regenerating fibres because of a lack of TLR activation. Consistent with our findings, mice deficient for MyD88 have been shown to have less myocardial inflammation and injury, reduced CVB3 viral titres, and improved survival [53].

Osteopontin, a multifunctional secreted glycophosphoprotein, has been shown to contribute to many physiological and pathological processes, such as immune responses, inflammation, and remodelling [23,54-56]. In the present study, we found that treatment with ssRNA significantly up-regulated $O P N$ mRNA expression and protein levels in $\mathrm{A} / \mathrm{J}$ muscles compared with saline-injected controls. Elevated expression of OPN has been detected in cardiotoxininjured muscle, the muscles of $m d x$ mice, and patients with DMD [40,57,58]. The up-regulation of OPN in the ssRNA-injected muscles of MyD88-sufficient mice, but not MyD88-deficient mice, also suggests that OPN is downstream of TLR-7 and -8. Up-regulation of OPN expression in injured muscle has previously been attributed to other cells in addition to immune cells such as macrophages and activated T-lymphocytes [24,58]. Therefore, it is likely that immune cells such as macrophages, natural killer cells, and neutrophils (as shown by positive staining with CD11b) may contribute in part to the OPN expression observed in the present study.

Based on these data, we hypothesize that leaky dysferlin-deficient skeletal muscle releases endogenous danger signals (eg ssRNA). These in turn bind to their respective TLRs (TLR-7/8) on muscle and immune cells and activate downstream processes, which result in the secretion of pro-inflammatory cytokines, such as OPN, that lead to degeneration, recruitment of inflammatory cells, and regeneration in the dysferlinopathic skeletal muscle (Figure 7). 
In conclusion, the results of this study provide a working model where unstable or poorly repaired myofibre membranes exude cytoplasmic components such as RNA that activate TLR-7/8 pathways. The precise mechanism by which the activation of endosomally located TLRs is unknown. The activation of innate immunity pathways further worsens the initial damage caused by the dysferlin gene defect. The loss of signalling ability by these TLRs, as a result of knocking out MyD88, appears not only to improve muscle function, but also to reduce inflammation and the secretion of pro-inflammatory cytokines, and therefore damage to the muscle fibres. Our study does not exclude a role for other TLR ligands (eg TLR-4) in the disease pathogenesis of dysferlinopathy. More studies are warranted to determine the precise contribution of TLRs to muscle damage, and additional studies are needed to determine the therapeutic potential of blocking this pathway in dysferlinopathy.

\section{Acknowledgments}

Dr Nagaraju is supported by NIH (1K26RR032082; 1P50AR060836-01; 1U54HD071601; 2R24HD050846 -06) and DOD grants (W81XWH-11-1-0330; W81 XWH-11-1-0782; W81XWH-10-1-0659; W81XWH11-1-0809; W81XWH-09-1-0599), and a translational research grant from MDA and pilot grant from PPMD. The funders had no role in the study design, data collection and analysis, decision to publish, or preparation of the manuscript. We would like to thank Dr Deborah McClellan for editing this manuscript.

\section{Author contribution statement}

$\mathrm{KU}, \mathrm{HC}, \mathrm{BA}, \mathrm{EPH}$, and $\mathrm{KN}$ conceived and designed the experiments. $\mathrm{KU}, \mathrm{HC}, \mathrm{BA}, \mathrm{AS}, \mathrm{SR}$, and JV performed the experiments. KU, BA, AS, TH, BW, $\mathrm{BC}, \mathrm{JQ}, \mathrm{KT}, \mathrm{EPH}$, and $\mathrm{KN}$ analysed the data. All authors were involved in writing the paper and had final approval of the submitted and published versions.

\section{References}

1. Liu J, Aoki M, Illa I, et al. Dysferlin, a novel skeletal muscle gene, is mutated in Miyoshi myopathy and limb girdle muscular dystrophy. Nature Genet 1998; 20: 31-36.

2. Bashir R, Britton S, Strachan T, et al. A gene related to Caenorhabditis elegans spermatogenesis factor fer- 1 is mutated in limb-girdle muscular dystrophy type 2B. Nature Genet 1998; 20: 37-42.

3. Anderson LV, Davison K, Moss JA, et al. Dysferlin is a plasma membrane protein and is expressed early in human development. Hum Mol Genet 1999; 8: 855-861.

4. Illa I, Serrano-Munuera C, Gallardo E, et al. Distal anterior compartment myopathy: a dysferlin mutation causing a new muscular dystrophy phenotype. Ann Neurol 2001; 49: 130-134.

5. De Luna N, Freixas A, Gallano P, et al. Dysferlin expression in monocytes: a source of mRNA for mutation analysis. Neuromuscul Disord 2007; 17: 69-76.
6. Ho M, Gallardo E, McKenna-Yasek D, et al. A novel, blood-based diagnostic assay for limb girdle muscular dystrophy 2B and Miyoshi myopathy. Ann Neurol 2002; 51: 129-133.

7. Nagaraju K, Rawat R, Veszelovszky E, et al. Dysferlin deficiency enhances monocyte phagocytosis: a model for the inflammatory onset of limb-girdle muscular dystrophy 2B. Am J Pathol 2008; 172: $774-785$.

8. Ampong $\mathrm{BN}$, Imamura $\mathrm{M}$, Matsumiya $\mathrm{T}$, et al. Intracellular localization of dysferlin and its association with the dihydropyridine receptor. Acta Myol 2005; 24: 134-144.

9. Huang Y, Laval SH, van Remoortere A, et al. AHNAK, a novel component of the dysferlin protein complex, redistributes to the cytoplasm with dysferlin during skeletal muscle regeneration. FASEB J 2007; 21: 732-742.

10. Klinge L, Laval S, Keers S, et al. From T-tubule to sarcolemma: damage-induced dysferlin translocation in early myogenesis. FASEB J 2007; 21: 1768-1776.

11. Bansal D, Miyake K, Vogel SS, et al. Defective membrane repair in dysferlin-deficient muscular dystrophy. Nature 2003; 423: 168-172.

12. Han R, Campbell KP. Dysferlin and muscle membrane repair. Curr Opin Cell Biol 2007; 19: 409-416.

13. Han R. Muscle membrane repair and inflammatory attack in dysferlinopathy. Skelet Muscle 2011; 1: 10.

14. Angelini C, Grisold W, Nigro V. Diagnosis by protein analysis of dysferlinopathy in two patients mistaken as polymyositis. Acta Myol 2011; 30: 185-187.

15. Amato AA, Brown RH Jr. Dysferlinopathies. Handb Clin Neurol 2011; 101: 111-118.

16. Vinit J, Samson M Jr, Gaultier JB, et al. Dysferlin deficiency treated like refractory polymyositis. Clin Rheumatol 2010; 29: 103-106.

17. Nguyen K, Bassez G, Krahn M, et al. Phenotypic study in 40 patients with dysferlin gene mutations: high frequency of atypical phenotypes. Arch Neurol 2007; 64: 1176-1182.

18. Rawat R, Cohen TV, Ampong B, et al. Inflammasome up-regulation and activation in dysferlin-deficient skeletal muscle. Am J Pathol 2010; 176: 2891-2900.

19. McNally EM, Ly CT, Rosenmann H, et al. Splicing mutation in dysferlin produces limb-girdle muscular dystrophy with inflammation. Am J Med Genet 2000; 91: 305-312.

20. Gallardo E, Rojas-Garcia R, de Luna N, et al. Inflammation in dysferlin myopathy: immunohistochemical characterization of 13 patients. Neurology 2001; 57: 2136-2138.

21. Klinge L, Aboumousa A, Eagle M, et al. New aspects on patients affected by dysferlin deficient muscular dystrophy. J Neurol Neurosurg Psychiatry 2010; 81: 946-953.

22. Denhardt DT, Guo X. Osteopontin: a protein with diverse functions. FASEB J 1993; 7: 1475-1482.

23. Denhardt DT, Noda M, O'Regan AW, et al. Osteopontin as a means to cope with environmental insults: regulation of inflammation, tissue remodeling, and cell survival. J Clin Invest 2001; 107: 10551061.

24. Vetrone SA, Montecino-Rodriguez E, Kudryashova E, et al. Osteopontin promotes fibrosis in dystrophic mouse muscle by modulating immune cell subsets and intramuscular TGF-beta. J Clin Invest 2009; 119: 1583-1594.

25. Pegoraro E, Hoffman EP, Piva L, et al. SPP1 genotype is a determinant of disease severity in Duchenne muscular dystrophy. Neurology 2011; 76: 219-226.

26. Uaesoontrachoon K, Yoo HJ, Tudor EM, et al. Osteopontin and skeletal muscle myoblasts: association with muscle regeneration and regulation of myoblast function in vitro. Int $J$ Biochem Cell Biol 2008; 40: 2303-2314. 
27. Shinohara ML, Lu L, Bu J, et al. Osteopontin expression is essential for interferon-alpha production by plasmacytoid dendritic cells. Nature Immunol 2006; 7: 498-506.

28. Shinohara ML, Jansson M, Hwang ES, et al. T-bet-dependent expression of osteopontin contributes to $\mathrm{T}$ cell polarization. Proc Natl Acad Sci U S A 2005; 102: 17101-17106.

29. Shinohara ML, Kim HJ, Kim JH, et al. Alternative translation of osteopontin generates intracellular and secreted isoforms that mediate distinct biological activities in dendritic cells. Proc Natl Acad Sci U S A 2008; 105: 7235-7239.

30. Zhang X, Kimura Y, Fang C, et al. Regulation of Toll-like receptormediated inflammatory response by complement in vivo. Blood 2007; 110: 228-236.

31. Pasare C, Medzhitov R. Control of B-cell responses by Toll-like receptors. Nature 2005; 438: 364-368.

32. St Paul M, Barjesteh N, Paolucci S, et al. Toll-like receptor ligands induce the expression of interferon-gamma and interleukin-17 in chicken CD4+ T cells. BMC Res Notes 2012; 5: 616.

33. Lerario A, Cogiamanian F, Marchesi C, et al. Effects of rituximab in two patients with dysferlin-deficient muscular dystrophy. BMC Musculoskelet Disord 2010; 11: 157.

34. Han R, Frett EM, Levy JR, et al. Genetic ablation of complement C3 attenuates muscle pathology in dysferlin-deficient mice. J Clin Invest 2010; 120: 4366-4374.

35. Farini A, Sitzia C, Navarro C, et al. Absence of $\mathrm{T}$ and $\mathrm{B}$ lymphocytes modulates dystrophic features in dysferlin deficient animal model. Exp Cell Res 2012; 318: 1160-1174.

36. Ho M, Post CM, Donahue LR, et al. Disruption of muscle membrane and phenotype divergence in two novel mouse models of dysferlin deficiency. Hum Mol Genet 2004; 13: 1999-2010.

37. Spurney CF, Gordish-Dressman H, Guerron AD, et al. Preclinical drug trials in the mdx mouse: assessment of reliable and sensitive outcome measures. Muscle Nerve 2009; 39: 591-602.

38. Rayavarapu S, Van der Meulen JH, Gordish-Dressman H, et al. Characterization of dysferlin deficient SJL/J mice to assess preclinical drug efficacy: fasudil exacerbates muscle disease phenotype. PLoS One 2010; 5: e12981.

39. Pfaffl MW. A new mathematical model for relative quantification in real-time RT-PCR. Nucleic Acids Res 2001; 29: e45.

40. Porter JD, Khanna S, Kaminski HJ, et al. A chronic inflammatory response dominates the skeletal muscle molecular signature in dystrophin-deficient mdx mice. Hum Mol Genet 2002; 11: 263-272.

41. Hoffman EP, Gordish-Dressman H, McLane VD, et al. Alterations in osteopontin modify muscle size in females in both humans and mice. Med Sci Sports Exerc 2013; 45: 1060-1068.

42. Tidball JG. Inflammatory processes in muscle injury and repair. Am J Physiol Regul Integr Comp Physiol 2005; 288: R345-R353.
43. Takeda K, Akira S. TLR signaling pathways. Semin Immunol 2004; 16: $3-9$.

44. Matzinger P. The danger model: a renewed sense of self. Science 2002; 296: 301-305.

45. Lennon NJ, Kho A, Bacskai BJ, et al. Dysferlin interacts with annexins $\mathrm{A} 1$ and $\mathrm{A} 2$ and mediates sarcolemmal wound-healing. $J$ Biol Chem 2003; 278: 50466-50473.

46. Kono H, Rock KL. How dying cells alert the immune system to danger. Nature Rev Immunol 2008; 8: 279-289.

47. Lai Y, Di Nardo A, Nakatsuji T, et al. Commensal bacteria regulate Toll-like receptor 3-dependent inflammation after skin injury. Nature Med 2009; 15: 1377-1382.

48. Chen YW, Nagaraju K, Bakay M, et al. Early onset of inflammation and later involvement of TGFbeta in Duchenne muscular dystrophy. Neurology 2005; 65: 826-834.

49. Cheung EV, Tidball JG. Administration of the non-steroidal antiinflammatory drug ibuprofen increases macrophage concentrations but reduces necrosis during modified muscle use. Inflamm Res 2003; 52: $170-176$.

50. Hodgetts S, Radley H, Davies M, et al. Reduced necrosis of dystrophic muscle by depletion of host neutrophils, or blocking $\mathrm{TNF} \alpha$ function with Etanercept in mdx mice. Neuromuscul Disord 2006; 16: 591-602.

51. Nguyen HX, Tidball JG. Interactions between neutrophils and macrophages promote macrophage killing of rat muscle cells in vitro. J Physiol 2003; 547: 125-132.

52. Pizza FX, Hernandez IJ, Tidball JG. Nitric oxide synthase inhibition reduces muscle inflammation and necrosis in modified muscle use. J Leukoc Biol 1998; 64: 427-433.

53. Fuse K, Chan G, Liu Y, et al. Myeloid differentiation factor- 88 plays a crucial role in the pathogenesis of Coxsackievirus B3induced myocarditis and influences type I interferon production. Circulation 2005; 112: 2276-2285.

54. Alchi B, Nishi S, Kondo D, et al. Osteopontin expression in acute renal allograft rejection. Kidney Int 2005; 67: 886-896.

55. Choi JS, Cha JH, Park HJ, et al. Transient expression of osteopontin mRNA and protein in amoeboid microglia in developing rat brain. Exp Brain Res 2004; 154: 275-280.

56. Scatena M, Liaw L, Giachelli CM. Osteopontin: a multifunctional molecule regulating chronic inflammation and vascular disease. Arterioscler Thromb Vasc Biol 2007; 27: 2302-2309.

57. Haslett JN, Sanoudou D, Kho AT, et al. Gene expression comparison of biopsies from Duchenne muscular dystrophy (DMD) and normal skeletal muscle. Proc Natl Acad Sci U S A 2002; 99: 1500015005.

58. Hirata A, Masuda S, Tamura $\mathrm{T}$, et al. Expression profiling of cytokines and related genes in regenerating skeletal muscle after cardiotoxin injection: a role for osteopontin. Am J Pathol 2003; 163: $203-215$.

\section{SUPPORTING INFORMATION ON THE INTERNET}

The following supporting information may be found in the online version of this article.

Supplementary Materials and Methods. Generation and genotyping of transgenic mice; western blotting of MyD88 protein; Illumina beadchip microarray. Additional microarray information: The original data is available in the NCBI's Gene Expression Omnibus public database (http://www.ncbi.nlm.nih.gov/geo/). GEO series accession number: GSE46420.

Figure S1. Generation of transgenic mouse models.

Figure S2. TLR signalling pathway identified by Ingenuity Pathway Analysis, obtained by comparing TA muscles of A/J mice that had been injected with ssRNA versus saline. 
Figure S3. TLR signalling pathway identified by Ingenuity Pathway Analysis, obtained by comparing TA muscles of WT and double-deficient mice injected with ssRNA versus saline.

Table S1. TLR-associated genes that were differentially expressed in TA muscles of WT, A/J, and double-deficient mice exposed to ssRNA, compared with those treated with saline.

Table S2. MyD88-associated genes that were differentially expressed in TA muscles of WT, A/J, and double-deficient mice exposed to ssRNA versus saline.

\section{Years ago in the Journal of Pathology...}

Observations upon the standardisation of bacterial vaccines by the Wright, the hæmocytometer, and the plate culture methods

Ernest Glynn, Mildred Powell, A. Armstrong Rees and G. Lissand Cox

Spontaneous and artificial development of giant cells in vitro

G. C. Weil

A new method of marking paraffin blocks for purposes of orientation or identification W. Henwood Harvey

To view these articles, and more, please visit: www.thejournalofpathology.com

Click 'ALL ISSUES (1892 - 2011)', to read articles going right back to Volume 1, Issue 1.

\section{The Journal of Pathology Understanding Disease}

